\title{
Mesoscale physical principles of collective cell organization
}

\author{
Xavier Trepat*1,2,3,4 and Erik Sahai*5 \\ ${ }^{1}$ Institute for Bioengineering of Catalonia, Barcelona 08028, Spain \\ ${ }^{2}$ Unitat de Biofísica i Bioenginyeria, Facultat de Medicina, Universitat de Barcelona, Barcelona 08036, Spain \\ ${ }^{3}$ Institució Catalana de Recerca i Estudis Avançats (ICREA), Barcelona 08010, Spain \\ ${ }^{4}$ Centro de Investigación Biomédica en Red en Bioingeniería, Biomateriales y Nanomedicina, Barcelona 08028, \\ Spain \\ ${ }^{5}$ The Francis Crick Institute, 1 Midland Road, London, NW1 1AT, UK. \\ *Corresponding authors: xtrepat@ibecbarcelona.eu, erik.sahai@crick.ac.uk
}


We review recent evidence showing that cell and tissue dynamics are governed by mesoscale physical principles. These principles can be understood in terms of simple state diagrams in which control variables include force, density, shape, adhesion, and self-propulsion. An appropriate combination of these physical quantities gives rise to emergent phenomena such as cell jamming, topological defects, and underdamped waves. Mesoscale physical properties of cell assemblies are found to precede and instruct biological functions such as cell division, extrusion, invasion and gradient sensing. These properties are related to properties of biomolecules, but cannot be predicted from biochemical principles. Thus, biological function is governed by emergent mesoscale states that can be predicted by a simple set of physical properties.

Most organs in the human body consist of sheets of cells termed epithelia ${ }^{1}$. During early development, epithelia are composed by a single cell layer that adopts a simple shape such as a hollow sphere or an ellipse. As development progresses epithelial sheets grow, differentiate, fold into complex three-dimensional shapes, and secrete their fibrous micro-environment -the extracellular matrix (ECM). Epithelial sheets exhibit complex dynamic behaviors that are not observed in single cells, neither can they be simply predicted from the observation of isolated cells $^{2-9}$. Understanding how the properties of sub-cellular structures relates to the complex physical properties of biological matter presents a compelling challenge $\mathrm{e}^{10-12}$. The advent of new microscopy technologies, such as super-resolution microscopy and correlative live cell and electron microscopy, is boosting our knowledge of nanoscale structures and interactions in cells ${ }^{13,14}$. Yet, the time and length scales at which tissue dynamics emerges remains largely unknown. Here we discuss mesoscale principles of collective cell organization. We begin by summarizing the main stress-bearing elements that form cells and tissues, and the main physical variables that determine their dynamics. We next review emergent phenomena in epithelial cell sheets and discuss theoretical frameworks that capture them in terms of simple integrative principles. Finally, we discuss how such principles might be applicable to more complex living tissues such as threedimensional assemblies.

\section{Subcellular determinants of collective cell dynamics}

In this section we summarize the main subcellular structures that cells use to generate, transmit and transduce physical forces. These are dynamic structures and organelles such as cytoskeletal filaments, the nucleus, cell-cell junctions and cell-ECM junctions.

The cytoskeleton. The structure of individual cells is governed by the cytoskeleton (Fig. 1). This is a network of three main classes of dynamic polymers. Actin filaments are continuously polymerizing and depolymerizing in a manner that consumes energy in the form of $\mathrm{ATP}^{15}$. Individual filaments are directional with actin monomers added at one end and detaching at the other. The rate of polymerization can be regulated by many factors and can lead to rates of filament growth in the order of several $\mu \mathrm{m} / \mathrm{min}^{16}$. In most migrating cells, actin monomer addition occurs at the front of the cell and generates a pushing force on the plasma membrane, which has a 
membrane tension of $200-400 \mathrm{pN} / \mu \mathrm{m}$. In some cells, all the force generated by polymerization is used to push the membrane forward, whereas in other cells actin polymerization at the membrane also leads to rearward movement of the filaments (this is termed retrograde flow). A range of proteins can modulate polymerization rates and nucleate new fibers from the side of existing ones; together with filament crosslinking and bundling proteins they enable the emergence of actin networks with complex geometries. Myosin motor proteins act as 'molecular machines' that utilize ATP to move along actin filaments and generate a few $\mathrm{pN}$ of force per stroke ${ }^{17}$. The dimeric nature of the predominant myosins in epithelial cells enables them to interact with two actin filaments at the same time, thereby generating contractile force ${ }^{18}$. This force can be transmitted to the plasma membrane via linker proteins enabling control of cell shape and, in some cases, internal hydrostatic pressure $^{19}$. Membrane spanning ion pumps can change the osmotic pressure balance of cells. Changes in cell shape and pressure can combine to change membrane tension, and several molecules exhibit tension dependent binding to membranes. The curved shape of some membrane binding linkers confers further complexity by favoring their binding to regions of the membrane with specific curvature ${ }^{20}$.

From a soft matter physics perspective, the actomyosin network can be treated as an active polar gel whose dynamics differ fundamentally from those of passive polymer gels in equilibrium ${ }^{21}$. Activity arises from the continuous consumption of free energy during polymerization and motor activity, which keeps the material far from thermodynamic equilibrium. Polarity arises from the asymmetric polymerization/depolymerization rates at each end of the filament. The active polar nature of the acto-myosin cytoskeleton enables remarkable non-equilibrium phenomena such as oscillations and waves in the absence of inertia ${ }^{22}$. While F-actin networks are highly complex at the nanometer scale, their cross-linking, myosin force generation, and perpetual turnover confer visco-elastic properties to the actin network ${ }^{23}{ }^{24}$. Indeed, different experimental approaches ${ }^{25}$ (Box 1) have successfully shown that the actin cytoskeleton behaves as a visco-elastic material at the micron scale. Viscoelasticity of the cytoskeleton should not be interpreted in terms of a finite number of time scales represented by a collection of springs and dashpots in series or parallel connection. Instead, extensive data now supports that time scales in the cytoskeleton are broadly distributed so that the elastic and loss moduli follow a weak power law in frequency ${ }^{26,27}$. These moduli are influenced positively both by polymerized actin and myosin activity.

Intermediate filaments are slightly larger in diameter than actin filaments, they are less dynamic and do not rely on ATP ${ }^{28},{ }^{29}$. Their mechanical response is strongly dependent on loading rate and their extensibility is partly determined by a structural switch from $\alpha$-helices to $\beta$-sheets ${ }^{30}$. These properties suggest that intermediate filaments protect cells from fast, large deformations ${ }^{31}$. Microtubules are the largest class of cytoskeletal filament. Similar to actin, they are polar filaments, but polymerization and de-polymerization occurs at the same filament end and their dynamics depends on GTP as an energy source ${ }^{32}$. Microtubule motor proteins transport cargo around the cell, including membrane vesicles, but do not generate significant contractile force. By contrast, microtubules can bear significant compressive load and, in some cases, this leads to 
buckling $^{33}$. In addition to their mechanical properties, microtubules are critical for the polarity of epithelial cells - that is the asymmetric delivery of cellular components to different of regions of the plasma membrane; either apical or basal in the non-migratory epithelial cells or front or rear in migrating cells. Stable cell polarity depends on motor proteins moving in a directed manner on microtubules delivering or receiving distinct 'cargos' from different regions of the cell ${ }^{34}$. Thus asymmetry in plasma membrane is linked to underlying asymmetry in the cytoskeleton. Cytoskeletal state and the movement of membranes is largely controlled by two classes of molecular 'switches' - RHO-GTPases and RAB-GTPases ${ }^{35}{ }^{36}$.

In addition to the cytoskeleton, the physical properties of individual cells are significantly influenced by the nucleus. This organelle is densely packed with DNA wrapped around histone protein complexes and encased by an envelope of intermediate filament proteins called lamins and a bi-layered membrane ${ }^{29}$. This nuclear envelope is connected to the cytoskeleton via LINC protein complexes. Lamins play a significant role in determining nuclear stiffness, that can vary between 0.1-10 $\mathrm{kPa}$ range ${ }^{37,38}$. Lamins act to protect the DNA from physical damage, but because the nucleus is the largest organelle in the cell they can also influence the overall cellular stiffness and the ability of cells to move through small gaps ${ }^{39}$.

Interactions with Neighbors and Substrates. The elements described above are important determinants of the internal physical properties of the cell. However, the behavior of cells in the context of a tissue depends on their interaction with other cells and the extracellular environment. A multitude of plasma membrane spanning molecules can mediate adhesion between cells. Cadherins are critical for epithelial cell cohesion through the formation of adherens junctions. In these junctions, cadherins are coupled to the actin cytoskeleton enabling actomyosin forces to be transmitted between cells ${ }^{40,41}$. This linkage to a contractile network means that junctions are typically under tension, with an individual cadherin junction withstanding 20-50pN before breaking ${ }^{42}$. Clustering of cadherins can increase the force needed to pull two cells apart into the range of $\mathrm{nN}^{43}$. The linkage of cadherins to the actin network is finely regulated by both internal and external mechanisms. If force is applied to cadherin-mediated junctions they can enhance coupling to the cytoskeleton and locally stiffen it ${ }^{44}$. Tight junctions perform a barrier function and enable the transport of ions across epithelial layers to be actively regulated. This can play an important role in the control of fluid pressures in tissues. Desmosomes are another class of cellcell junction. They are coupled with intermediate filaments and the resulting supra-cellular network confers mechanical resilience on cell layers ${ }^{45}$. Together, adherens junctions, desmosomes, and tight junctions are the major mediators or epithelial cell-cell adhesion and their regulation enables emergent behaviors in cell sheets that are not observed in single cell systems.

Most cells also make contact with the supporting matrix that confers long-lasting form to tissues. This matrix consists of a variety of protein polymers, including collagens and laminins. Like many biological polymers, they exhibit strain-stiffening behavior and can be extensively cross-linked ${ }^{46}$. The latter process typically increases the bulk modulus and results in elastic behavior. A subset of matrix proteins are heavily glycosylated and their hydration can provide a gel-like property to the 
extra-cellular matrix. A large variety of cell surface proteins interact with the matrix and these interactions are critical for both tissue structure and cell migration. The integrin family of heterodimers are central to cell-matrix interactions and are capable of resisting forces in the range $20-100 \mathrm{pN}$, although most engaged integrins in a cell are subjected to forces $<7 \mathrm{pN}^{47}$. The affinity of the heterodimer can be regulated from the inside of the cell, and, conversely, the engagement of external matrix ligands can alter the inter-cellular conformation of integrins leading to changes in cell signaling. Further, integrins frequently cluster into micron scale assemblies called focal adhesions that are capable of exerting forces around $25 \mathrm{nN}^{48}$. The intra-cellular part of integrins is coupled to the actin cytoskeleton via a range of adaptor molecules ${ }^{49}$. This linkage is important for actin polymerization to exert a pushing force on the plasma membrane. The coupling of actin filaments to integrin-mediated adhesions is not constant. Several of the adaptor proteins that link actin and integrins undergo force-induced conformation changes that alter their binding properties. This leads to two important emergent features. First, there is a clutch between actin and integrins that modulates how effectively actin polymerization pushes the cell membrane. Second, when force is applied on integrins it is transmitted through $\alpha$-helical domains to the interior of the cell where it can change the conformation of proteins bound to integrins. For example, talin and p130Cas undergo conformational changes when subjected to forces in the low pN range ${ }^{50}$, which influences their ability to interact with other protein partners and signal to the actin cytoskeleton leading to local increases in actin polymerization. The change of internal cell properties, such as F-actin dynamics, in response to external mechanical cues has led integrin-mediated adhesions to be termed 'mechano-responsive's1.

\section{Emergent mechanics of cell monolayers}

Despite the broad diversity of subcellular components described above, collective cell dynamics is ultimately determined by a limited set of key mechanical properties. In the spirit of soft matter physics, current efforts attempt to identify these properties and provide a coarse-grained description of the laws that govern collective cell dynamics (Box 2, Fig. 2). The focus of this type of approach is not so much to identify how one specific molecule might affect one specific physical property, but rather to explain collective tissue dynamics in terms of state diagrams that capture the phenomenology on the basis of a small set of key variables. This set includes, but is not limited to, cell-matrix traction, intercellular stress and self-propulsion.

Key mechanical variables. A traction is a force per unit area applied at any surface of a cell ${ }^{52}$. In the context of cell mechanics, the traction vector is understood as the force per unit area applied by the cell on its surrounding inert microenvironment. In general, cells exert traction by pulling on focal adhesions using their actomyosin machinery, but cells lacking integrins and even passive liquid drops are able to exert significant traction ${ }^{53}$, 54 . Several approaches have been described to quantify cell tractions in 2D and 3D by measuring deformations of materials surrounding cells ${ }^{55}$ (Box 1). The combination of force measurements with imaging has revealed that the generation of traction is a highly dynamic process in which the force generated by myosin causes continuous 
binding/unbinding events of integrins and accessory proteins. As a consequence, actin near adhesion sites is usually seen to move retrogradely towards the center of the cell ${ }^{56,57}$.

Tractions at cell-cell interfaces are usually not called tractions but adhesion forces. In the simple case of a suspended cell doublet in which the cytoskeleton localizes mainly at the cell surface called the cell cortex- adhesion forces are parallel to the cell-cell interface ${ }^{58}$. In general, force at any point of a cell-cell junction has a component parallel to the cell-cell interface (shear component) and one normal to it (normal component) $)^{59}$. Like cell-matrix traction sites, cell-cell adhesion sites are highly dynamic and adhesion proteins turn-over frequently.

If the cell is studied at a scale larger than the cytoskeletal mesh, then it can be treated as a continuum (Box 2). At any point of this continuum the mechanical state of the cell is fully captured by the stress tensor, which defines the force per unit area applied on any surface centered at any point of a tissue. In general, stress has a normal component and a shear component ${ }^{5}$. The normal component indicates the extent to which a material is tensed or compressed at a given point, whereas the shear component indicates forces parallel to the surface.

Besides the ability to generate active stresses, a key feature that differentiates tissues from passive soft materials is the self-propulsive ability of each individual cell. Cells achieve self-propulsion by extending distinct types of protrusions and adhering them to their surroundings. The two main types of protrusions are thin actin rich extensions called filopodia or lamellipodia, and spherical membrane blisters called blebs. Cells migrate using lamellipodia, filopodia, or blebs depending on a diversity of intrinsic and extrinsic variables including expression of adhesion proteins, density and composition of the extracellular matrix, confinement, topology, and cortical contractility ${ }^{60,61}$. In order to migrate, cells adhere their protrusions to the surrounding matrix either specifically through membrane receptors such as integrins, or unspecifically using frictional interactions.

Even at the level of the single isolated cell, the relationship between forces exerted by cells and their velocity is not straightforward. For example, a cell crawling on a flat substrate generates tractions that are orders of magnitude smaller than the force needed to propel their body through the surrounding viscous fluid ${ }^{62}$. Traction forces are thus not generated to achieve the function of migration, but rather to actively probe and adhere to the microenvironment. They may also be crucial for leukocytes to exit the blood and home to areas of tissue damage in the face of high shear forces exerted by blood flow ${ }^{63}$. As discussed above, mechanical sensing of the substrate depends on force-driven conformational changes in proteins within cell-matrix adhesion complexes ${ }^{64}$. The substrate stiffness threshold at which these changes occur is tuned by the binding rates of integrins to the underlying substrate ${ }^{65}$. Nonetheless, traction and migration are not independent because the flow of actin in a protrusion is inversely proportional to the traction generated by that protrusion ${ }^{56}$.

Collective cell dynamics. Despite being genetically identical, cells in monolayers exhibit heterogeneous properties in terms of shape, adhesion, and dynamics. For example, cells close to a free edge are usually large, flat, protrusive and able to generate large traction forces ${ }^{66}$. Conversely, cells behind the edge tend to be cuboidal and to generate low traction forces through cryptic 
lamellipodia protruded underneath their neighbors ${ }^{67}$ (Fig. 1). Within these two categories, there exist large variations as well. Some cells at the leading edge are much larger, protrusive, and motile than their immediate neighbors, which has led to the idea that collective migration of monolayers is driven by "leader" cells ${ }^{68}$. In the bulk of the monolayer, some cells also have distinctive dynamic signatures such as the ability to rotate in swirls ${ }^{69}$. The dynamics of these swirls is determined by intrinsic and extrinsic factors such as cell adhesion, division and confinement ${ }^{70}$.

The renewed interest in cell monolayers -an experimental system that dates back more than one century- originates from the development of new technologies to measure not only velocity and deformation fields but also tractions and intercellular stresses (Box 1) ${ }^{25}$. Force mapping has unveiled phenomenological principles of cell organization such as the alignment of the cell body with the direction of maximum stress ${ }^{5,71,72}$. This phenomenon, called plithotaxis, implies that cells organize in sheets so as to minimize intercellular shear stress. Plithotaxis provides a mechanim for cells to migrate collectively in a preferred mechanical direction during wound healing and cancer invasion $^{73}$.

The organization of cells in sheets and clusters has also been shown to enable collective sensing of both chemical and mechanical gradients ${ }^{4,74}$ (Fig. 3). When cells are prevented from transmitting forces by disrupting cell-cell junctions or by inhibiting myosin motors, collective gradient sensing is often impaired. Another striking emergent phenomenon in cell monolayers is their ability to propagate mechanical waves. In response to sudden unconfinement, the first row of cells at the monolayer edge spreads and migrates towards the freely available substrate, whereas cells behind remain static ${ }^{66,75}$. With time, every cell row becomes progressively engaged in collective motion following a wave of deformation and force generation. Propagation of mechanical waves has now been observed in confined clusters ${ }^{76,77}$, in expanding colonies ${ }^{66,75}$, and in colliding monolayers ${ }^{78}$ (Fig. 3). Propagation of mechanical waves in inertial matter is a trivial physical phenomenon that can be simply explained by an exchange of potential and kinetic energy. In cell monolayers, which lack inertia, alternative mechanisms must be at play to introduce second derivatives with respect to time and provide an effective inertia ${ }^{76}$. This could be achieved through the interplay between cell mechanics and molecular circuits involving mechanotransduction, such as those involving the mechanosensing protein merlin, which links forces at cell-cell junctions with the regulation of the protrusive and force-generating action of RHO-GTPases, ${ }^{6,79}$.

A general feature of cell monolayers -and possibly one that is intuitive to any cell biologist who has ever performed cell culture- is that cells growing on a dish slow down their motion as cell density increases ${ }^{80}$. From a physical perspective, this behavior is reminiscent to that of granular materials such as sand or coffee beans close to a jamming transition; as system density increases, each constitutive element becomes trapped by its neighbors, the energy required for structural rearrangements rises, and the system transitions from a fluid to a disordered solid ${ }^{81}$. Careful analysis of cell velocity fields in proliferating cell monolayers showed that the analogy between the behavior of cell monolayers and granular materials close to a jamming transition is deeper than expected. Like particles in granular materials, cells in dense monolayers move in large groups whose length scale grows with cell density ${ }^{5}$. Moreover, cells in a monolayer exhibit dynamic heterogeneities in cell migration, a non-Arrhenius dependence of relaxation times on cell density, 
peaks in the vibrational density of states, and a shift in the position of the four-point susceptibility function ${ }^{80}$.

A perhaps less intuitive observation is that fluid to solid transitions in cell monolayers can also occur at constant density. This striking result was first predicted by theoretical analysis of vertex models $^{82}$ (Box 3). These models aim at capturing the dynamics of tissues in terms of the motion of vertices representing junctions between three or more neighboring cells ${ }^{83}$. A key ingredient behind the success of vertex models is that they readily enable neighbor exchanges. In the absence of mitosis and extrusion, neighbor exchanges in monolayers occur through $\mathrm{T} 1$ transitions, whereby one edge between two cells shrinks until vanishing and a new edge is created at the same point between two different cells. $\mathrm{Bi}$ et $\mathrm{al}^{82}$ identified that the energy distribution of $\mathrm{T} 1$ transitions in a vertex model is a function of a dimensionless geometric factor called target shape index $p_{o}=$ $P_{o} / \sqrt{A_{o}}$, where $P_{o}$ and $A_{o}$ are preferred perimeter and area, respectively. For regular polygons, $p_{o}$ increases with the number of sides, with $p_{o}=3.72$ for a regular hexagon and $p_{o}=3.81$ for a regular pentagon. More strikingly, they found that below a critical value $p_{o}=3.81$, energy barriers are finite and the system behaves like a jammed solid. By contrast, above $p_{o}=3.81$ energy barriers become vanishingly small and the monolayer behaves like an unjammed liquid. This result, which has now been extended to 3D bulk tissues ${ }^{84}$, suggested that cell monolayers exhibit a jamming transition controlled by a geometric parameter independent of tissue density. This prediction was successfully tested using bronchial epithelial cells maturing in air-liquid interface ${ }^{85}$. As maturation time increased, $p_{o}$ decreased without a noticeable change in cell density, and monolayer dynamics slowed down. Remarkably, bronchial epithelial cells from asthmatic donors exhibited higher values of $p_{o}$ and remained unjammed for a longer time than cells obtained from healthy donors.

The ability of early 2D vertex models to precisely capture the jamming transition in cell monolayers is remarkable taking into account that they did not include the ability of cells to exert traction forces on their substrate or to self-propel their body. One approach to include selfpropulsion to the description of 2D monolayers was the development of self-propelled Voronoi Models (SPVs, Box 3) ${ }^{86-88}$. These models predict four relevant states in monolayers defined in terms of the shape index $p_{o}$ and the characteristic time of alignment between neighbors $J$ (Fig. 3). These states are solid, liquid, solid flock and liquid flock ${ }^{86,87}$. The solid (or jammed) phase emerges at low $p_{o}$ and low $J$, and it is characterized by the absence of cell rearrangements and of global tissue movement. If $p_{o}$ is increased at low values of $J$, the monolayer is predicted to enter a liquid phase in which the spatial correlation length remains low but local rearrangements become frequent. These two phases are analogous to those predicted by $2 \mathrm{D}$ vertex models in the absence of self-propulsion or with self-propulsion but in the absence of alignment ${ }^{86-88}$. At higher values of $J$, the SPV predicts the emergence of flocking, this is, an increase of the spatial correlation length of the velocity field. Monolayer flocks can be solid or liquid. In the flocking solid state, the monolayer flows cohesively without neighbor exchanges. By contrast, in the flocking liquid state the monolayer combines long-range correlation with frequent local rearrangements (Fig. 3). Experimental observations of transitions between solid (jammed) and liquid phases have been reported in maturing bronchial epithelial cells at the air-liquid interface ${ }^{85}$. A transition between non-flocking and flocking liquid was recently observed in response to overexpression of the 
endocytic GTPase RAB5A ${ }^{86}$. These examples illustrate how differentiation processes or expression changes in one single gene can be rationalized in terms of transitions in a physical phase diagram.

Dynamics of cell monolayers has also been interpreted in terms of active nematics ${ }^{2,}$, 9 . Like molecules in liquid crystals, cells conforming a dense monolayer are polarized and often exhibit an elongated shape. In addition, they are able to self-propel their body, which gives rise to a range of phenomena inaccessible to inert liquid crystals such as long-range order, giant fluctuations, unattenuated wave propagation, and turbulence at low Reynolds numbers ${ }^{89}$. A common feature of liquid crystals is the presence of singular points in the orientational field, known as topological defects. In contrast with passive systems, topological defects in active systems can form and annihilate spontaneously. Dynamic topological defects have been reported in cell monolayers of elongated shape, such as fibroblasts ${ }^{2}$, but also in cell types that are not elongated but have a polarized cytoskeleton such as MDCK cells. In the latter case, cells located at topological defects were shown to display higher probability to be extruded from the monolayer ${ }^{3}$. The origin of extrusion was proposed to be the compressive stress experienced by cells as a consequence of the topological configuration at the defect site. This phenomenon illustrates how the collective dynamics of a monolayer can precede and instruct biological function.

\section{Scaling up to three-dimensional assemblies}

While some biological tissues can be considered essentially as two-dimensional structures, with only low levels of curvature relative to the scale of the cell (for example, the skin excluding hair follicles), the majority of tissues have complex three-dimensional shapes. How the behaviors articulated above in 2D systems determine 3D architecture is poorly understood, but much effort is being directed at this problem. The simplest experimental models involve spheroids of cells that can be either solid or hollow. We will discuss both of these before concluding with remarks on higher levels of topological complexity found in some tissues.

Solid spheroids. The simplest three-dimensional form that a group of cells can adopt is a sphere, but even this form has additional complexity compared to simple monolayers. The distance of a cell from the sphere's surface affects its access to nutrients and oxygen. This imposes a biochemical gradient not observed in 2D systems and, in many cases, can limit the size to which the sphere can grow ${ }^{90,91}$. Cell movement within a spheroid could mitigate against this by enabling cells to move in and out of nutrient/oxygen limited areas, but this has not been explored in any detail. Indeed, much less is known about the migratory behavior of cells in spheroids. Unlike 2D systems where all cells have contact with the underlying matrix substrate, cells in the interior of the spheroid only contact other cells or the small amounts of matrix proteins on the surface of other cells. Consistent with this, both adherens junctions and desmosomal connections have been shown to play a key role in cells migrating in 3D multi-cellular structures ${ }^{92}$. Frictional mechanisms of traction are also likely to play a more significant role in migration in confined environments. In such contexts, frictional traction stresses have been measured in the Pa range ${ }^{93}$. However, frictional mechanisms can, in principle, generate significantly higher stresses. The movement of one cell requires the displacement of its neighbors and the strength of cell-cell adhesion, shape, and deformability of the surrounding cells all influence the ability of a cell to move and its coordination 
with neighbors ${ }^{94,95}$. Strong cell-cell adhesion and low deformability typically lead to low levels of movement within spheroids or jamming. The transitions from jammed to un-jammed states in 3D are less well understood than in 2D systems, but the same principles are likely to apply with both a shape factor $p_{o}$ and alignment rate $J$ being critical. The outer layer of cells does contact the matrix that supports the spheroid and can exert traction forces in a similar manner to 2D cell systems. Experimental observation reveals that in some cases there is sufficient coordination to generate rotational behaviors ${ }^{96}$. The mechanisms underlying the emergence of these behaviors remain incompletely understood, but the analysis of the flocking liquid and solid states in 2D systems is likely to be relevant. Rotational behavior is not just a curiosity of simple 3D cell systems but is linked to the development of more complex shapes. In Drosophila the egg chamber is initially spherical, but the rotational movement of the outer cell layer influences the underlying matrix structure and is required for the prolate shape of the egg to develop ${ }^{97,98}$.

Pressure can also be an important influence of cell behavior in 3D systems. Unless spheroids are growing in aqueous suspension, in which case the fluid pressure is negligible, the matrix that supports the spheroid will exhibit some resistance to deformation. The conversion of soluble nutrients to biomass as a result of cell growth then requires some deformation of the surrounding matrix to accommodate the change in volume. This is not uniform throughout the spheroid, but is greater in the center and can feedback upon the growth rate of cells ${ }^{99}$. This can ultimately limit the growth of spheroids or require biological remodeling of the surrounding matrix to generate space for cell growth. Pressure in spheroids and tumours has been estimated in the range 1-40 $\mathrm{kPa}^{100,101}$.

Hollow spheroids. Many epithelial cells have the ability to form fluid filled spheres and more complex branched structures. Key to this behavior are cell-cell adhesions mediated by cadherins and cell polarity that is stabilized by microtubules. Cells in the nascent sphere in contact with the supporting matrix receive signals that polarize the cytoskeleton. Microtubules and RAB-GTPases coordinate the delivery of specialized membrane to the part of the cell away from the basal surface ${ }^{102}$. This generates a specialized apical membrane in the interior, and the action of ion pumps and the death of cells not in contact with the substrate leads to a hollow sphere. Cells in a hollow sphere can exhibit jammed or unjammed behaviors similar to 2D systems. Cell-cell junctions ensure that fluid flow between cells in an epithelium is tightly regulated. Ion pumps can regulate the osmotic pressure of the fluid within a spheroid and thereby modulate its rate of expansion ${ }^{103}$. Consideration of the bulk tissue properties therefore requires to take into account the inter-luminal or interstitial pressure. In developed organs with a lumen, such as the mammary gland, the pressure can exceed $10^{4} \mathrm{~Pa}^{104}$, whereas in vitro estimations in monolayered semi-spheres are on the order of $100 \mathrm{~Pa}^{105}$. Luminal pressure $(\Delta \mathrm{P}$ in Fig. $2 \mathrm{~h})$ depends on coordinated cell polarization to ensure that solutes are transported in a consistent direction by all the cells in the structure, and it is linked to interfacial tension and epithelial curvature through Laplace's law. In line with this idea, numerous studies have shown that the loss of key cell polarity genes, many of which being tumor suppressor genes, prevents the formation of ordered hollow spheroids. Loss of polarity therefore contributes causally to the disordered nature of tumors and the recurrent observation that tumor cells form solid, not hollow, spheroids. 
Multiple Cell Types and Complex geometries. Both normal tissues and tumors contain a mixture of cell types. This adds a further layer of complexity to the mesoscale properties of the tissue. The jamming/unjamming transitions of cell layers described above are sensitive to the introduction of small numbers of cells with distinctive properties, such as altered cell shape. Even relatively few elongated cells, such as fibroblasts, are predicted to perturb jamming. Cells with different cell-cell adhesion properties may also sort themselves into distinct regions. Simple models predict that cells with low cell-cell adhesion are preferentially sorted to the exterior of spheroids ${ }^{106}$. However, more detailed analysis suggests that this mechanism may not be sufficient and different tension at the boundaries between cell types and their jamming state may also play a role $e^{58,107,108}$. Experimental studies have confirmed the importance of myosin for the formation of sharp tissue boundaries ${ }^{109}$.

Differences in substrate traction forces and actin protrusion dynamics will also add complexity. Highly protrusive fibroblasts with strong traction forces are better able to move into the matrix that surrounds spheroids, thus acting as leader cells ${ }^{110}$. These lead to the emergence of cell strands leaving the spheroid and generates complex 'stellate' 3D shapes ${ }^{111}$. Leaders and followers impact each other's function continuously ${ }^{112}$; leaders pull on followers and open gaps in the ECM to enable collective invasion ${ }^{111}$ whereas followers contribute to polarize leaders and to steer their migration $^{111,113}$.

The majority of epithelial tissues are not spherical but highly folded as this benefits fitting a large surface area (functionality) into a small volume. Our understanding of the emergence of the geometries found in tissues such as the lungs, intestine, brain or mammary gland is still in its infancy; however, there are two areas that are intensively studied at the moment. The first is tissue folding. Physically, tissue folding can be understood as a mechanical instability originating from excess compressive stress in a specific layer of a tissue ${ }^{114}$. One possible mechanism for this process involves differential contraction or expansion of apical and basal membranes in 2D systems leading to curvature. 2D vertex modelling is being adapted to $3 \mathrm{D}$ geometries to provide a theoretical framework for this process (Box 2). The tractability of these models is appealing and they are likely to be relevant when folding mechanisms lead to the generation of hollow tubes ${ }^{7,8}$. However, the emergence of complex tissues typically happens early in embryogenesis when the small scale of the organism means that curvature is typically high. Thus, while these models could be powerful for explaining some of the early folding events in embryogenesis (when the surface curvature of the whole embryo is relatively low), they may be less suited to explaining how a complex tissue arises from a very small number of progenitor cells. Insights into how a complex structure emerges from a low number of cells in a highly curved structure are coming from organoid cultures ${ }^{115}$. In these systems, a small number of progenitor cells initially form a hollow spheroid and this then becomes branched or 'multi-lobed'. Non-uniformity in the ability of cells to expand or proliferate could lead to the preferential expansion of some regions of the spheroid that then become the nascent branches. Differential levels of proliferation and differentiation of specific tissue layers with respect to their surrounding has also been shown to trigger mechanical instabilities that give rise to the characteristic folded structures of diverse tissues such as brain ${ }^{116}$, lung and gut ${ }^{117,118}$. High pressure of the luminal fluid could further assist expansion and 
branching ${ }^{103}$. The origin and biophysical nature of the non-uniformity is not clear; it could relate to regions of jamming or increased contractility. Consistent with the latter idea, the growing ends of branches in mammary organoids are associated with a lack of contractile myoepithelial cells ${ }^{119}$. Folding and patterning associated with the initiation of hair follicles arise from compressive stresses caused by contraction of the epidermis by the dermis ${ }^{120}$. Imaging has also revealed that mammary cells in expanding branching regions exchange position very frequently and are not jammed. Further analysis has revealed that stochastic branching events followed by linear branch growth, and branch termination in the event of collision can explain the complex higher order patterns of branching observed in adult tissues collision ${ }^{115}$.

\section{Concluding remarks}

Understanding the transition from a single cell fertilized egg into the complex three-dimensional forms of multi-cellular organisms remains one of the great problems in all science. Principles from soft matter physics such as cell jamming and active nematics have yielded many new insights into the generation of organized multicellular structures. The rich phenomenology of cohesive tissues is increasingly well-captured by theoretical frameworks such as those based on vertex models ${ }^{82}$,

${ }^{83}$, particle models ${ }^{121,122}$, or Potts models ${ }^{123}$. Moreover, several new approaches now enable the accurate quantification of the key physical variables that govern tissue dynamics. In relatively simple tissues such as cell monolayers, we are currently at a point where theory and experiments can be confronted and feedback each other closely. Despite these exciting advances, important challenges remain. Further work is needed to understand how the molecular structure and dynamics of biomolecules confers distinct physical properties on cell-cell and cell-matrix interactions. It will also be crucial to enhance our understanding of three-dimensional systems and the feedback between cell-driven changes in the extra-cellular matrix, cell adhesions and cell state. To achieve this goal, models will need to incorporate tissue-matrix interactions. Finally, new tools are needed to probe tissue mechanics in systems of increasing complexity such as embryos or regenerating tissues.

Physicists and biologists have long collaborated to develop quantitative tools to probe living systems, but they have also regarded each other with skepticism. Physicists have traditionally considered that living systems are far too messy to be understood in terms of a simple set of unifying laws. Biologists, by contrast, have considered that physical approaches are far too abstract to capture cells and tissues in the broadest diversity and specificity. These views are rapidly changing. Physicists now understand that there is new physics in living matter, and that there is no better laboratory than the living cell to study the physics of systems far from thermodynamic equilibrium. Conversely, biologists are increasingly regarding physics not only as a toolbox to extract much-needed quantitative information, but also as a source of new conceptual frameworks that explain and predict the behavior of living systems at multiple length scales. Physics and biology are thus merging into a unified discipline to provide an integrative understanding of active living matter that captures its diversity and specificity as well as its fundamental laws. 


\section{ACKNOWLEDGEMENTS}

We apologize to the many colleagues whose work could not be cited owing to space constraints. We thank Ernest Latorre, Fabio Giavazzi, Roberto Cerbino, Guillaume Jacquemet, Johanna Ivaska Justin Taraska, and Kem Sochacki for contributing original materials, and all members of our laboratories for critical comments and encouragement. The authors acknowledge support by the Spanish Ministry of Economy, Industry and Competitiveness through the Centro de Excelencia Severo Ochoa Award to the Institute of Bioengineering of Catalonia (XT) and through grant BFU2015-65074-P (XT), the Generalitat de Catalunya (Cerca Program and 2014-SGR-927 to XT), the European Research Council (CoG-616480 to XT), and the European Commission (project 731957 to XT). ES is funded by the Francis Crick Institute, which receives its core funding from Cancer Research UK (FC001144), the UK Medical Research Council (FC001144), and the Wellcome Trust (FC001144). 


\section{REFERENCES}

1. Alberts, B. et al. Molecular Biology of the Cell, Edn. 6th Edition (Garland Science, 2016).

2. Duclos, G., Erlenkämper, C., Joanny, J.-F. \& Silberzan, P. Topological defects in confined populations of spindle-shaped cells. Nature Physics 13, 58-62 (2016).

3. Saw, T.B. et al. Topological defects in epithelia govern cell death and extrusion. Nature 544, 212216 (2017).

4. Sunyer, R. et al. Collective cell durotaxis emerges from long-range intercellular force transmission. Science (New York, N.Y 353, 1157-1161 (2016).

5. Tambe, D.T. et al. Collective cell guidance by cooperative intercellular forces. Nat Mater (2011).

6. Das, T. et al. A molecular mechanotransduction pathway regulates collective migration of epithelial cells. Nature cell biology 17, 276-287 (2015).

7. Hogan, B.L. \& Kolodziej, P.A. Organogenesis: molecular mechanisms of tubulogenesis. Nat Rev Genet 3, 513-523 (2002).

8. Bryant, D.M. \& Mostov, K.E. From cells to organs: building polarized tissue. Nature reviews 9, 887901 (2008).

9. Kawaguchi, K., Kageyama, R. \& Sano, M. Topological defects control collective dynamics in neural progenitor cell cultures. Nature 545, 327-331 (2017).

10. Spurlin, J.W., 3rd \& Nelson, C.M. Building branched tissue structures: from single cell guidance to coordinated construction. Philosophical transactions of the Royal Society of London 372 (2017).

11. Laurent, J. et al. Convergence of microengineering and cellular self-organization towards functional tissue manufacturing. Nature Biomedical Engineering 1, 939-956 (2017).

12. Ladoux, B. \& Mege, R.M. Mechanobiology of collective cell behaviours. Nature reviews 18, 743757 (2017).

13. Kanchanawong, P. et al. Nanoscale architecture of integrin-based cell adhesions. Nature 468, 580584 (2010).

14. Chen, B.C. et al. Lattice light-sheet microscopy: imaging molecules to embryos at high spatiotemporal resolution. Science (New York, N.Y 346, 1257998 (2014).

15. Pollard, T.D. \& Borisy, G.G. Cellular motility driven by assembly and disassembly of actin filaments. Cell 112, 453-465 (2003).

16. Loisel, T.P., Boujemaa, R., Pantaloni, D. \& Carlier, M.F. Reconstitution of actin-based motility of Listeria and Shigella using pure proteins. Nature 401, 613-616 (1999).

17. Finer, J.T., Simmons, R.M. \& Spudich, J.A. Single myosin molecule mechanics: piconewton forces and nanometre steps. Nature 368, 113-119 (1994).

18. Newell-Litwa, K.A., Horwitz, R. \& Lamers, M.L. Non-muscle myosin II in disease: mechanisms and therapeutic opportunities. Dis Model Mech 8, 1495-1515 (2015).

19. Mitchison, T.J., Charras, G.T. \& Mahadevan, L. Implications of a poroelastic cytoplasm for the dynamics of animal cell shape. Seminars in cell \& developmental biology 19, 215-223 (2008).

20. McDonald, N.A. \& Gould, K.L. Linking up at the BAR: Oligomerization and F-BAR protein function. Cell Cycle 15, 1977-1985 (2016).

21. Kruse, K., Joanny, J.F., Julicher, F., Prost, J. \& Sekimoto, K. Generic theory of active polar gels: a paradigm for cytoskeletal dynamics. Eur Phys J E Soft Matter 16, 5-16 (2005).

22. Jülicher, F. \& Prost, J. Spontaneous Oscillations of Collective Molecular Motors. Physical review letters 78, 4510-4513 (1997).

23. Joanny, J.F. \& Prost, J. Active gels as a description of the actin-myosin cytoskeleton. HFSP J 3, 94104 (2009).

24. Alvarado, J., Sheinman, M., Sharma, A., MacKintosh, F.C. \& Koenderink, G.H. Force percolation of contractile active gels. Soft Matter 13, 5624-5644 (2017). 
25. Roca-Cusachs, P., Conte, V. \& Trepat, X. Quantifying forces in cell biology. Nature cell biology 19, 742-751 (2017).

26. Fabry, B. et al. Scaling the microrheology of living cells. Physical review letters 87, 148102 (2001).

27. Alcaraz, J. et al. Microrheology of human lung epithelial cells measured by atomic force microscopy. Biophysical journal 84, 2071-2079 (2003).

28. Sanghvi-Shah, R. \& Weber, G.F. Intermediate Filaments at the Junction of Mechanotransduction, Migration, and Development. Front Cell Dev Biol 5, 81 (2017).

29. Goldmann, W.H. Intermediate filaments and cellular mechanics. Cell Biol Int (2017).

30. Block, J. et al. Nonlinear Loading-Rate-Dependent Force Response of Individual Vimentin Intermediate Filaments to Applied Strain. Physical review letters 118, 048101 (2017).

31. Beil, M. et al. Sphingosylphosphorylcholine regulates keratin network architecture and viscoelastic properties of human cancer cells. Nature cell biology 5, 803-811 (2003).

32. Roostalu, J. \& Surrey, T. Microtubule nucleation: beyond the template. Nature reviews 18, 702710 (2017).

33. Brangwynne, C.P. et al. Microtubules can bear enhanced compressive loads in living cells because of lateral reinforcement. The Journal of cell biology 173, 733-741 (2006).

34. Muroyama, A. \& Lechler, T. Microtubule organization, dynamics and functions in differentiated cells. Development (Cambridge, England) 144, 3012-3021 (2017).

35. Haga, R.B. \& Ridley, A.J. Rho GTPases: Regulation and roles in cancer cell biology. Small GTPases 7, 207-221 (2016).

36. Pfeffer, S.R. Rab GTPases: master regulators that establish the secretory and endocytic pathways. Molecular biology of the cell 28, 712-715 (2017).

37. Physical Sciences - Oncology Centers, N. et al. A physical sciences network characterization of nontumorigenic and metastatic cells. Scientific reports 3, 1449 (2013).

38. Lee, J.S. et al. Nuclear lamin A/C deficiency induces defects in cell mechanics, polarization, and migration. Biophysical journal 93, 2542-2552 (2007).

39. Guilak, F., Tedrow, J.R. \& Burgkart, R. Viscoelastic properties of the cell nucleus. Biochemical and biophysical research communications 269, 781-786 (2000).

40. Takeichi, M. Dynamic contacts: rearranging adherens junctions to drive epithelial remodelling. Nature reviews 15, 397-410 (2014).

41. Ladoux, B., Nelson, W.J., Yan, J. \& Mege, R.M. The mechanotransduction machinery at work at adherens junctions. Integr Biol (Camb) 7, 1109-1119 (2015).

42. Panorchan, P. et al. Single-molecule analysis of cadherin-mediated cell-cell adhesion. Journal of cell science 119, 66-74 (2006).

43. Shawky, J.H. \& Davidson, L.A. Tissue mechanics and adhesion during embryo development. Developmental biology 401, 152-164 (2015).

44. Yonemura, S., Wada, Y., Watanabe, T., Nagafuchi, A. \& Shibata, M. alpha-Catenin as a tension transducer that induces adherens junction development. Nature cell biology 12, 533-542 (2010).

45. Hatzfeld, M., Keil, R. \& Magin, T.M. Desmosomes and Intermediate Filaments: Their Consequences for Tissue Mechanics. Cold Spring Harb Perspect Biol 9 (2017).

46. Storm, C., Pastore, J.J., MacKintosh, F.C., Lubensky, T.C. \& Janmey, P.A. Nonlinear elasticity in biological gels. Nature 435, 191-194 (2005).

47. Chang, A.C. et al. Single Molecule Force Measurements in Living Cells Reveal a Minimally Tensioned Integrin State. ACS Nano (2016).

48. Balaban, N.Q. et al. Force and focal adhesion assembly: a close relationship studied using elastic micropatterned substrates. Nature cell biology 3, 466-472 (2001).

49. Winograd-Katz, S.E., Fassler, R., Geiger, B. \& Legate, K.R. The integrin adhesome: from genes and proteins to human disease. Nature reviews 15, 273-288 (2014). 
50. Yao, M. et al. Mechanical activation of vinculin binding to talin locks talin in an unfolded conformation. Scientific reports 4, 4610 (2014).

51. Sun, Z., Guo, S.S. \& Fassler, R. Integrin-mediated mechanotransduction. The Journal of cell biology 215, 445-456 (2016).

52. Dembo, M. \& Wang, Y.L. Stresses at the cell-to-substrate interface during locomotion of fibroblasts. Biophysical journal 76, 2307-2316. (1999).

53. Bastounis, E. et al. Both contractile axial and lateral traction force dynamics drive amoeboid cell motility. The Journal of cell biology 204, 1045-1061 (2014).

54. Murrell, M.P. et al. Liposome adhesion generates traction stress. Nature Physics 10, 163-169 (2014).

55. Schwarz, U.S. \& Soine, J.R. Traction force microscopy on soft elastic substrates: A guide to recent computational advances. Biochimica et biophysica acta 1853, 3095-3104 (2015).

56. Chan, C.E. \& Odde, D.J. Traction dynamics of filopodia on compliant substrates. Science (New York, N.Y 322, 1687-1691 (2008).

57. Elosegui-Artola, A. et al. Mechanical regulation of a molecular clutch defines force transmission and transduction in response to matrix rigidity. Nature cell biology 18, 540-548 (2016).

58. Maître, J.-L. et al. Adhesion functions in cell sorting by mechanically coupling the cortices of adhering cells. Science (New York, N.Y 338, 253-256 (2012).

59. Maruthamuthu, V., Sabass, B., Schwarz, U.S. \& Gardel, M.L. Cell-ECM traction force modulates endogenous tension at cell-cell contacts. Proceedings of the National Academy of Sciences of the United States of America 108, 4708-4713 (2011).

60. Tozluoglu, M. et al. Matrix geometry determines optimal cancer cell migration strategy and modulates response to interventions. Nature cell biology 15, 751-762 (2013).

61. Tozluoglu, M., Mao, Y., Bates, P.A. \& Sahai, E. Cost-benefit analysis of the mechanisms that enable migrating cells to sustain motility upon changes in matrix environments. J R Soc Interface 12 (2015).

62. Bergert, M. et al. Force transmission during adhesion-independent migration. Nature cell biology 17, 524-529 (2015).

63. Yeh, Y.T. et al. Three-dimensional forces exerted by leukocytes and vascular endothelial cells dynamically facilitate diapedesis. Proceedings of the National Academy of Sciences of the United States of America 115, 133-138 (2018).

64. del Rio, A. et al. Stretching single talin rod molecules activates vinculin binding. Science (New York, N.Y 323, 638-641 (2009).

65. Elosegui-Artola, A. et al. Rigidity sensing and adaptation through regulation of integrin types. Nature Materials 13, 631-637 (2014).

66. Serra-Picamal, X. et al. Mechanical waves during tissue expansion. Nat Phys 8, 628-634 (2012).

67. Farooqui, R. \& Fenteany, G. Multiple rows of cells behind an epithelial wound edge extend cryptic lamellipodia to collectively drive cell-sheet movement. Journal of cell science 118, 51-63 (2005).

68. Reffay, M. et al. Interplay of RhoA and mechanical forces in collective cell migration driven by leader cells. Nature cell biology 16, 217-223 (2014).

69. Rossen, N.S., Tarp, J.M., Mathiesen, J., Jensen, M.H. \& Oddershede, L.B. Long-range ordered vorticity patterns in living tissue induced by cell division. Nat Commun 5, 5720 (2014).

70. Marel, A.K. et al. Flow and diffusion in channel-guided cell migration. Biophysical journal 107, 1054-1064 (2014).

71. Zaritsky, A. et al. Seeds of Locally Aligned Motion and Stress Coordinate a Collective Cell Migration. Biophysical journal 109, 2492-2500 (2015).

72. Theveneau, E. et al. Collective chemotaxis requires contact-dependent cell polarity. Developmental cell 19, 39-53 (2010). 
73. Trepat, X. \& Fredberg, J.J. Plithotaxis and emergent dynamics in collective cellular migration. Trends in cell biology 21, 638-646 (2011).

74. Malet-Engra, G. et al. Collective cell motility promotes chemotactic prowess and resistance to chemorepulsion. Curr Biol 25, 242-250 (2015).

75. Taeseok Daniel, Y., Hyun, K., Changhyeong, Y., Seung-Kuk, B. \& Kyoung, J.L. Collective pulsatile expansion and swirls in proliferating tumor tissue. New Journal of Physics 18, 103032 (2016).

76. Notbohm, J. et al. Cellular Contraction and Polarization Drive Collective Cellular Motion. Biophysical journal 110, 2729-2738 (2016).

77. Deforet, M., Hakim, V., Yevick, H.G., Duclos, G. \& Silberzan, P. Emergence of collective modes and tri-dimensional structures from epithelial confinement. Nat Commun 5, 3747 (2014).

78. Rodriguez-Franco, P. et al. Long-lived force patterns and deformation waves at repulsive epithelial boundaries. Nat Mater 16, 1029-1037 (2017).

79. Tlili, S. et al. in ArXiv e-prints, Vol. 1610 (2016).

80. Angelini, T.E. et al. From the Cover: Glass-like dynamics of collective cell migration. Proceedings of the National Academy of Sciences of the United States of America 108, 4714-4719 (2011).

81. Liu, A.J. \& Nagel, S.R. Nonlinear dynamics: Jamming is not just cool any more. Nature 396, 21-22 (1998).

82. Bi, D., Lopez, J.H., Schwarz, J.M. \& Manning, M.L. A density-independent rigidity transition in biological tissues. Nature Physics 11, 1074-1079 (2015).

83. Farhadifar, R., Roper, J.C., Aigouy, B., Eaton, S. \& Julicher, F. The influence of cell mechanics, cellcell interactions, and proliferation on epithelial packing. Curr Biol 17, 2095-2104 (2007).

84. Merkel, M. \& Manning, M.L. A geometrically controlled rigidity transition in a model for confluent 3D tissues. New Journal of Physics 20, 022002 (2018).

85. Park, J.A. et al. Unjamming and cell shape in the asthmatic airway epithelium. Nat Mater (2015).

86. Malinverno, C. et al. Endocytic reawakening of motility in jammed epithelia. Nat Mater 16, 587596 (2017).

87. Giavazzi, F. et al. in ArXiv e-prints, Vol. 1706 (2017).

88. Bi, D., Yang, X., Marchetti, M.C. \& Manning, M.L. Motility-driven glass and jamming transitions in biological tissues. Phys Rev $X \mathbf{6}$ (2016).

89. Marchetti, M.C. et al. Hydrodynamics of soft active matter. Reviews of Modern Physics 85, 11431189 (2013).

90. Carlsson, J. et al. The influence of oxygen on viability and proliferation in cellular spheroids. Int J Radiat Oncol Biol Phys 5, 2011-2020 (1979).

91. Conger, A.D. \& Ziskin, M.C. Growth of mammalian multicellular tumor spheroids. Cancer research 43, 556-560 (1983).

92. Ewald, A.J. et al. Mammary collective cell migration involves transient loss of epithelial features and individual cell migration within the epithelium. Journal of cell science 125, 2638-2654 (2012).

93. Charras, G. \& Sahai, E. Physical influences of the extracellular environment on cell migration. Nature reviews 15, 813-824 (2014).

94. Vedula, S.R. et al. Emerging modes of collective cell migration induced by geometrical constraints. Proceedings of the National Academy of Sciences of the United States of America 109, 1297412979 (2012).

95. Kabla, A.J. Collective cell migration: leadership, invasion and segregation. J R Soc Interface 9, 32683278 (2012).

96. Wang, H., Lacoche, S., Huang, L., Xue, B. \& Muthuswamy, S.K. Rotational motion during threedimensional morphogenesis of mammary epithelial acini relates to laminin matrix assembly. Proceedings of the National Academy of Sciences of the United States of America 110, 163-168 (2013). 
97. Cetera, M. et al. Epithelial rotation promotes the global alignment of contractile actin bundles during Drosophila egg chamber elongation. Nat Commun 5, 5511 (2014).

98. Chen, D.Y., Crest, J. \& Bilder, D. A Cell Migration Tracking Tool Supports Coupling of Tissue Rotation to Elongation. Cell reports 21, 559-569 (2017).

99. Dolega, M.E. et al. Cell-like pressure sensors reveal increase of mechanical stress towards the core of multicellular spheroids under compression. Nat Commun 8, 14056 (2017).

100. Stylianopoulos, T. et al. Causes, consequences, and remedies for growth-induced solid stress in murine and human tumors. Proceedings of the National Academy of Sciences of the United States of America 109, 15101-15108 (2012).

101. Stylianopoulos, T. The Solid Mechanics of Cancer and Strategies for Improved Therapy. J Biomech Eng 139 (2017).

102. Stenmark, H. Rab GTPases as coordinators of vesicle traffic. Nature reviews 10, 513-525 (2009).

103. Jaffe, A.B., Kaji, N., Durgan, J. \& Hall, A. Cdc42 controls spindle orientation to position the apical surface during epithelial morphogenesis. The Journal of cell biology 183, 625-633 (2008).

104. Peaker, M. The effect of raised intramammary pressure on mammary function in the goat in relation to the cessation of lactation. J Physiol 301, 415-428 (1980).

105. Tanner, C., Frambach, D.A. \& Misfeldt, D.S. Transepithelial transport in cell culture. A theoretical and experimental analysis of the biophysical properties of domes. Biophysical journal 43, 183-190 (1983).

106. Foty, R.A. \& Steinberg, M.S. The differential adhesion hypothesis: a direct evaluation. Developmental biology 278, 255-263 (2005).

107. Hutson, M.S., Brodland, G.W., Yang, J. \& Viens, D. Cell sorting in three dimensions: topology, fluctuations, and fluidlike instabilities. Physical review letters 101, 148105 (2008).

108. Pawlizak, S. et al. Testing the differential adhesion hypothesis across the epithelial-mesenchymal transition. New Journal of Physics 17, 083049 (2015).

109. Monier, B., Pelissier-Monier, A., Brand, A.H. \& Sanson, B. An actomyosin-based barrier inhibits cell mixing at compartmental boundaries in Drosophila embryos. Nature cell biology 12, 60-69 (2010).

110. Gaggioli, C. et al. Fibroblast-led collective invasion of carcinoma cells with differing roles for RhoGTPases in leading and following cells. Nature cell biology 9, 1392-1400 (2007).

111. Labernadie, A. et al. A mechanically active heterotypic E-cadherin/N-cadherin adhesion enables fibroblasts to drive cancer cell invasion. Nature cell biology 19, 224-237 (2017).

112. Theveneau, E. \& Linker, C. Leaders in collective migration: are front cells really endowed with a particular set of skills? F1000Res 6, 1899 (2017).

113. Weber, G.F., Bjerke, M.A. \& Desimone, D.W. A Mechanoresponsive Cadherin-Keratin Complex Directs Polarized Protrusive Behavior and Collective Cell Migration. Developmental cell (2011).

114. Nelson, C.M. On Buckling Morphogenesis. J Biomech Eng 138, 021005 (2016).

115. Hannezo, E. et al. A Unifying Theory of Branching Morphogenesis. Cell 171, 242-255 e227 (2017).

116. Tallinen, T. et al. On the growth and form of cortical convolutions. Nature Physics 12, 588-593 (2016).

117. Hannezo, E., Prost, J. \& Joanny, J.F. Instabilities of monolayered epithelia: shape and structure of villi and crypts. Physical review letters 107, 078104 (2011).

118. Shyer, A.E. et al. Villification: how the gut gets its villi. Science (New York, N.Y 342, 212-218 (2013).

119. Ewald, A.J., Brenot, A., Duong, M., Chan, B.S. \& Werb, Z. Collective epithelial migration and cell rearrangements drive mammary branching morphogenesis. Developmental cell 14, 570-581 (2008).

120. Shyer, A.E. et al. Emergent cellular self-organization and mechanosensation initiate follicle pattern in the avian skin. Science (New York, N.Y 357, 811-815 (2017). 
121. Basan, M., Elgeti, J., Hannezo, E., Rappel, W.J. \& Levine, H. Alignment of cellular motility forces with tissue flow as a mechanism for efficient wound healing. Proceedings of the National Academy of Sciences of the United States of America 110, 2452-2459 (2013).

122. Smeets, B. et al. Emergent structures and dynamics of cell colonies by contact inhibition of locomotion. Proceedings of the National Academy of Sciences of the United States of America 113, 14621-14626 (2016).

123. Graner, F. \& Glazier, J.A. Simulation of biological cell sorting using a two-dimensional extended Potts model. Physical review letters 69, 2013-2016 (1992).

124. Wang, N., Butler, J.P. \& Ingber, D.E. Mechanotransduction across the cell surface and through the cytoskeleton. Science (New York, N.Y 260, 1124-1127 (1993).

125. Campas, O. et al. Quantifying cell-generated mechanical forces within living embryonic tissues. Nat Methods 11, 183-189 (2014).

126. Colombelli, J. et al. Mechanosensing in actin stress fibers revealed by a close correlation between force and protein localization. Journal of cell science 122, 1665 (2009).

127. Kumar, S. et al. Viscoelastic retraction of single living stress fibers and its impact on cell shape, cytoskeletal organization, and extracellular matrix mechanics. Biophysical journal 90, 3762-3773 (2006).

128. Thoumine, O. \& Ott, A. Time scale dependent viscoelastic and contractile regimes in fibroblasts probed by microplate manipulation. Journal of cell science 110 ( Pt 17), 2109-2116 (1997).

129. Liang, C.C., Park, A.Y. \& Guan, J.L. In vitro scratch assay: a convenient and inexpensive method for analysis of cell migration in vitro. Nature protocols 2, 329-333 (2007).

130. Gardel, M.L. et al. Elastic behavior of cross-linked and bundled actin networks. Science (New York, N.Y 304, 1301-1305 (2004).

131. Kroy, K. \& Glaser, J. The glassy wormlike chain. New J. Phys., 416 (2007).

132. Rauzi, M., Verant, P., Lecuit, T. \& Lenne, P.F. Nature and anisotropy of cortical forces orienting Drosophila tissue morphogenesis. Nature cell biology 10, 1401-1410 (2008).

133. Blanch-Mercader, C. et al. Effective viscosity and dynamics of spreading epithelia: a solvable model. Soft Matter 13, 1235-1243 (2017).

134. Sochacki, K.A. \& Taraska, J.W. Correlative Fluorescence Super-Resolution Localization Microscopy and Platinum Replica EM on Unroofed Cells. Methods in molecular biology 1663, 219-230 (2017).

135. O'Rourke, K.P., Dow, L.E. \& Lowe, S.W. Immunofluorescent Staining of Mouse Intestinal Stem Cells. Bio Protoc 6 (2016).

136. Blanch-Mercader, C. \& Casademunt, J. Hydrodynamic instabilities, waves and turbulence in spreading epithelia. Soft Matter 13, 6913-6928 (2017).

137. Szabo, B. et al. Phase transition in the collective migration of tissue cells: experiment and model. Physical review. E, Statistical, nonlinear, and soft matter physics 74, 061908 (2006). 


\section{BOX 1: Measuring cell mechanics}

1) ATOMIC FORCE MICROSCOPY involves placing a cantilever of known deformability against a cell or other material. Known forces are applied and the deflection of the cantilever is measured, usually by reflecting light on $\mathrm{it}^{27}$.

2) BEAD DISPLACEMENT ASSAYS typically involve coating a bead with a biologically adhesive molecule and then placing it against a cell ${ }^{26}$. Depending on the type of bead, force can be applied by the use of magnetic fields or optical tweezers. A modification of the magnetic method allows repeated twisting of the bead, termed magnetic twisting cytometry ${ }^{124}$. This method allows for repeated interrogation of the cell's properties, enabling to determine whether the cell changes its mechanical properties in response to a mechanical challenge.

3) INSERT DEFORMATION assays involve the introduction of inserts (typically soft polymer gel spheres or lipid droplets) of known deformability into either a cell or a multi-cellular tissue $^{125}$. Forces within the cell or tissue can be interpreted from the insert deformation, especially if they are anisotropic and lead to a loss of sphericity.

4) LASER CUTTING assays involve the use of a high power focused laser to 'cut' a cellular structure or tissue. The recoil of the surrounding tissue can be used to infer the tension that the cut region was under ${ }^{126,127 .}$

5) TIMELAPSE MICROSCOPY involves repeated imaging of the cells to generate a movie of their motion. The movie can be analyzed by tracking individual cells, although at high cell density Particle Imaging Velocimetry is often used because the reliable identification and tracking of individual cells may not be possible. Genetically encoded fluorophores are used to label either specific cells or specific proteins within cells.

6) TRACTION FORCE MICROSCOPY involves growing cells on an elastic substrate of known deformability. The spatial deformation of the substrate by the cell is measured with a microscope (usually with the aid of fiducial fluorescent beads embedded in the substrate). Forces can be inferred by comparing the deformed and relaxed states of the substrate ${ }^{52}$.

7) MICROPLATE ASSAYS involve plating cells on a deformable substrate that is attached between one fixed plate and another that can be moved. This enables uniaxial compression or stretching to be performed. The response of the cell is typically studied by timelapse microscopy ${ }^{31,128}$.

8) WOUND HEALING ASSAYS rely on damaging an epithelial sheet using a laser or direct mechanical disruption, and the response is frequently monitored using a combination of the techniques listed above, including TIMELAPSE MICROSCOPY to obtain dynamics, LASER CUTTING, TRACTION FORCE MICROSCOPY, and MICROPLATE ASSAYS. They are particularly useful because, in conditions of tissue homeostasis, there is relatively little motion of epithelial cells except for that resulting from the birth of new cells or cell death. Perturbation of the homeostatic state therefore reveals regulatory mechanisms ${ }^{129}$. 


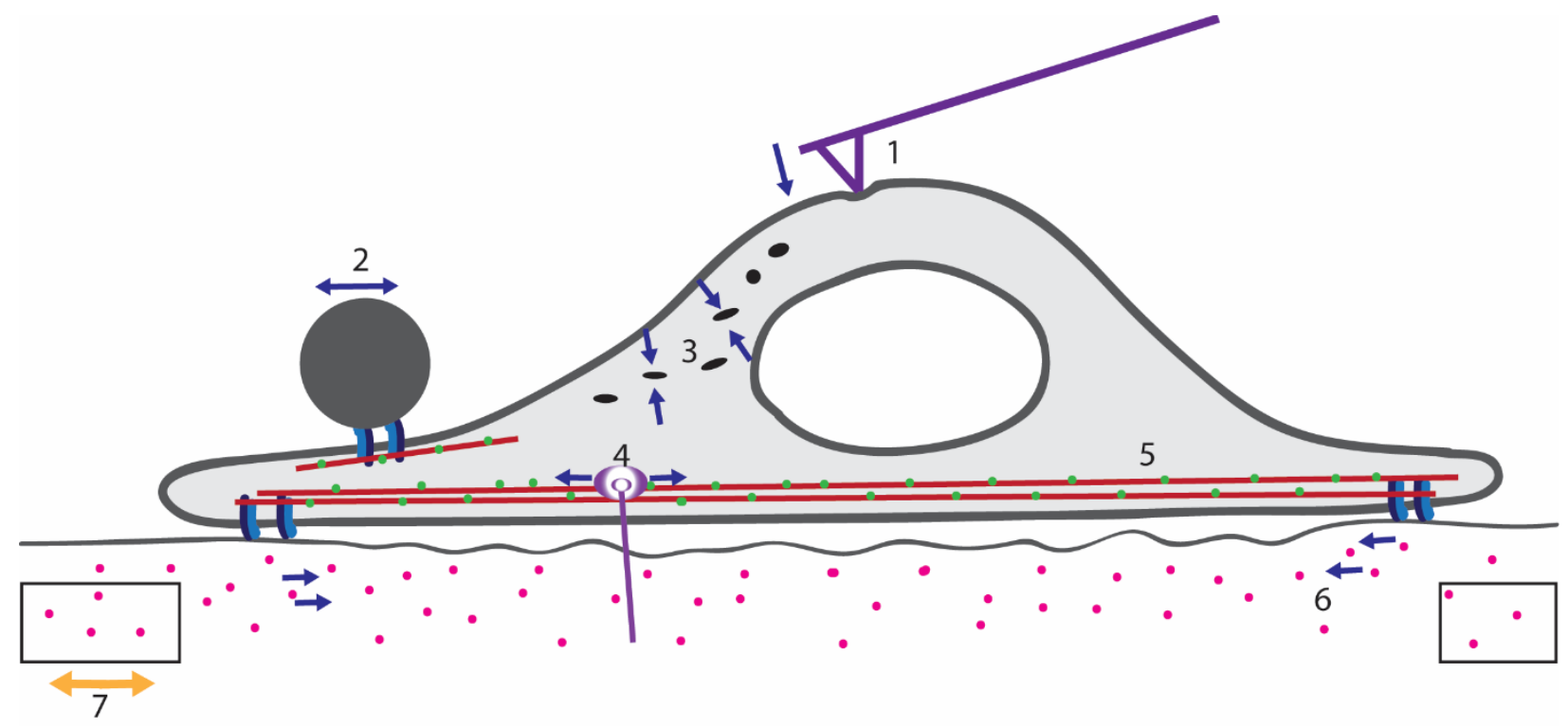

Figure Box 1: Image shows a cell on a deformable substrate with F-actin fibers in red, integrins in two-tone blue, and the cell nucleus in white. Green dots indicate fluorescent labelling of F-actin common in time-lapse analysis, pink dots indicate beads used for tracking substrate deformation, and dark grey ellipses are deformable inserts. Numbers highlight the measurement techniques described above. 


\section{BOX 2: Mechanobiology as a multiscale problem}

One the great challenges of mechanobiology is the existence of multiple length scales at which relevant mechanisms are at play. This problem has led to the development of diverse types of modelling approaches describing biological tissues at different scales. Examples of mechanical models of cells and tissues include the following.

1) CLUTCH MODELS describe the dynamics of cell-matrix adhesion at the molecular level (a,d). They take into account the rigidity of the matrix, the binding/unbinding $\left(k_{o n}, k_{\text {off }}\right)$ rates of adhesion proteins, and the force that myosin motors exert on these proteins through the actin cytoskeleton. Clutch models have been successful at predicting traction forces and dynamics of the leading edge of a protruding cell, sensing of rigidity and matrix ligand spacing, and durotaxis ${ }^{4,56,57 .}$

2) POLYMER NETWORK MODELS are based on the polymeric nature of the cytoskeleton $(\mathbf{c}, \mathbf{e})$. They take into account the properties of single filaments, the deformability and binding rates of polymer crosslinking proteins, and the activity of molecular motors that pull on the filaments. These models capture fundamental rheological properties of cells such as strainstiffening, scale-free elastic modulus, fluidization, and aging ${ }^{130,131}$.

3) VERTEX MODELS treat cell monolayers as 2D or 3D polygonal objects that share common vertices (see also BOX 3) (c,e). In 3D, these models are governed by tissue geometry and by surface tensions of the apico-basal $\left(\gamma_{a b}\right)$ and lateral surfaces $\left(\gamma_{1}\right)$. Vertex models have predicted dynamic aspects of monolayers such as their ability to deform through intercalation, to jam at constant density and to flock as solids and liquids ${ }^{82,83,86,87,132}$.

4) CONTINUUM MODELS describe tissues as active materials in which the cellular structure is coarse-grained $(\mathbf{d}, \mathbf{f})$. These models are formulated in terms of continuum vectorial and tensorial fields such as velocity $\vec{v}$, polarity $\vec{p}$ and stress $\sigma$. These variables are linked through viscosity $\eta$, elasticity $k$, and friction $\zeta$. In $3 \mathrm{D}$ they also incorporate luminal pressure $\triangle P$. Continuum models have successfully described complex features of cell monolayers such as wave propagation in the absence of inertia ${ }^{76,133}$. 

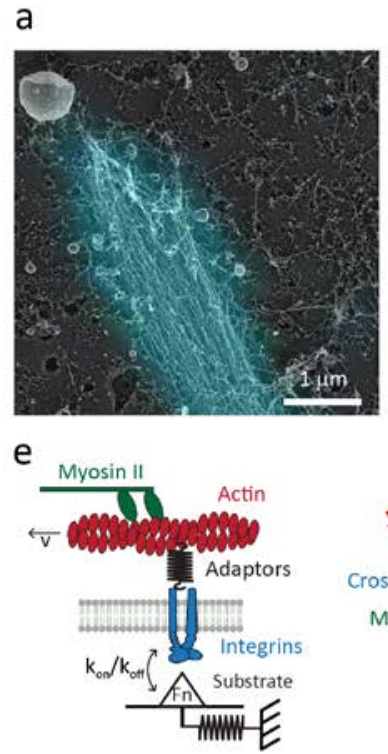

b

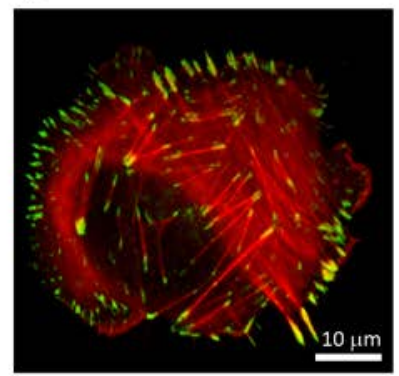

$f$

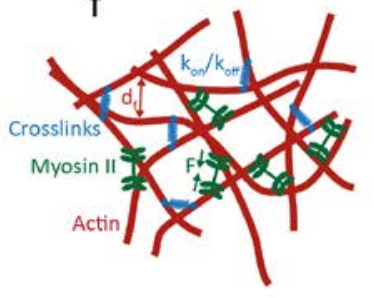

C

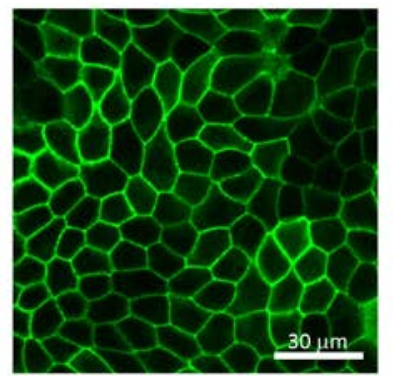

g

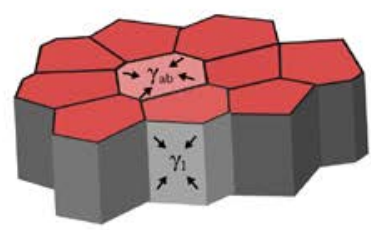

d

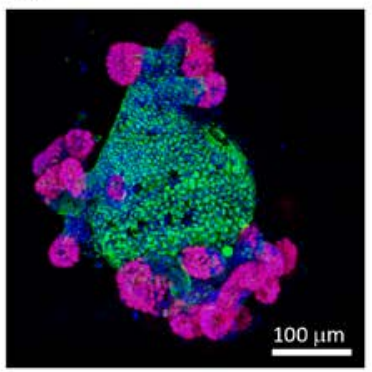

h

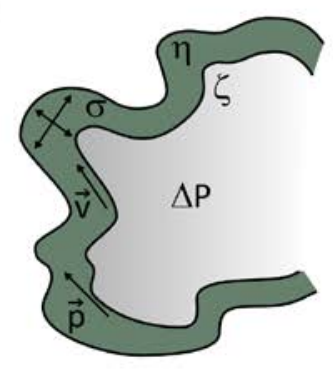

Figure Box 2. Mechanical structures and models of cells and tissues at different length scales.

(a) A focal adhesion of an unroofed Hela cell stained with fluorescent phalloidin (cyan) and imaged with TIRF/platinum replica TEM (grey) CLEM ${ }^{134}$. Image courtesy of J. Taraska and K. Sochacki. (b) An osteosarcoma cell (U2OS) plated on fibronectin. Actin (red) was imaged using STED and a focal adhesion marker (paxillin, green) was imaged using confocal microscopy. Image courtesy of G. Jacquemet and J. Ivaska. (c) A monolayer of epithelial cells in which actin the cell membrane was labelled using CAAX-GFP (green). (d) Immunofluorescent image of a small intestinal organoid showing markers of proliferation (EdU, Red), differentiation (Krt20, Green), and nuclei (blue). Adapted from reference ${ }^{135}$ (e) Clutch model. (f) Polymer network model. (g) 3D vertex model. (h) Continuum model. 


\section{BOX 3: Vertex models}

A variety of models have been recently implemented to explain the rich phenomenology of cell monolayers $^{79,121-123,136}$. Among such models, vertex models treat the cell monolayer as a closepacked mosaic of polygonal objects that represent constituent cells ${ }^{82,83}$. These models study the dynamic evolution of the 2D network formed by cell vertices and by connections between them. The position $x_{i}$ of each vertex $k$ is determined by the equation of motion

$$
\frac{d x_{k}}{d t}=\mu F_{k}
$$

where $F_{k}$ is the force acting on the vertex and $1 / \mu$ is the friction between the cell and its substrate. $F_{k}$ may be specified explicitly as a sum of contractile, adhesive, and osmotic contributions, or obtained implicitly by computing the gradient of an energy function of the monolayer. A common approach to compute such energy function is to express the energy of a given cell $i$ in terms of its area $A_{i}$ and its perimeter $P_{i}$ :

$$
E_{\text {monolayer }}=\sum_{i} E_{i}=\sum_{i} K_{A i}\left(A_{i}-A_{i 0}\right)^{2}+K_{P i}\left(P_{i}-P_{i 0}\right)^{2}
$$

The first term represents an area elasticity where $A_{i 0}$ is a preferred cell area and $K_{A i}$ is an area elastic modulus. This contribution to the cell energy can be understood in terms of cell incompressibility and resistance to height fluctuations. The second term represents a perimeter elasticity, with a preferred perimeter $P_{i 0}$ and a perimeter elastic modulus $K_{A i}$. Expansion of this second terms yields a quadratic term in $P_{i}$, which describes the contractility of the cell cortex, and a linear term in $P_{i}$, which reflects a competition between effective cell-cell adhesion and cortical tension. In this interpretation, cell-cell adhesion tends to increase the length of the edges, whereas cortical tension tends to shorten them.

To incorporate the self-propulsion, the formulation of vertex models has been combined with terms derived from particle models to give rise to self-propelled Voronoi models (SPV) ${ }^{86,87}$. Rather than focusing on the dynamics cell vertices, SPVs describe the motion of the center of each cell obtained by Voronoi Tesselation. The energy function describing the interaction between cells is the same than in vertex models but motion of the cell center is determined not only by the gradient of the energy function but also by a self-propulsion term $v_{0} \boldsymbol{n}_{\boldsymbol{k}}$. The equation of motion then reads:

$$
\frac{d \boldsymbol{x}_{\boldsymbol{k}}}{d t}=\mu \boldsymbol{F}_{\boldsymbol{k}}+v_{0} \boldsymbol{n}_{\boldsymbol{k}}
$$

where $\boldsymbol{n}_{\boldsymbol{k}}$ is a unit vector indicating the polarity of the cell and $v_{0}$ is a constant. In the simplest approximation, the polarization dynamics is governed by random rotational diffusion independent of the velocity of the cell and, therefore, of the movement of its neighbors ${ }^{88}$. However, dynamics of monolayers is better captured by imposing an active feedback mechanism inspired by flocking models ${ }^{137}$ that tends to align cell polarity with cell velocity. Given that cell velocity is in part due to the force exerted by the neighbors, this feedback mechanism effectively couples the movement of adjacent cells within an alignment rate $J^{86,87}$. 


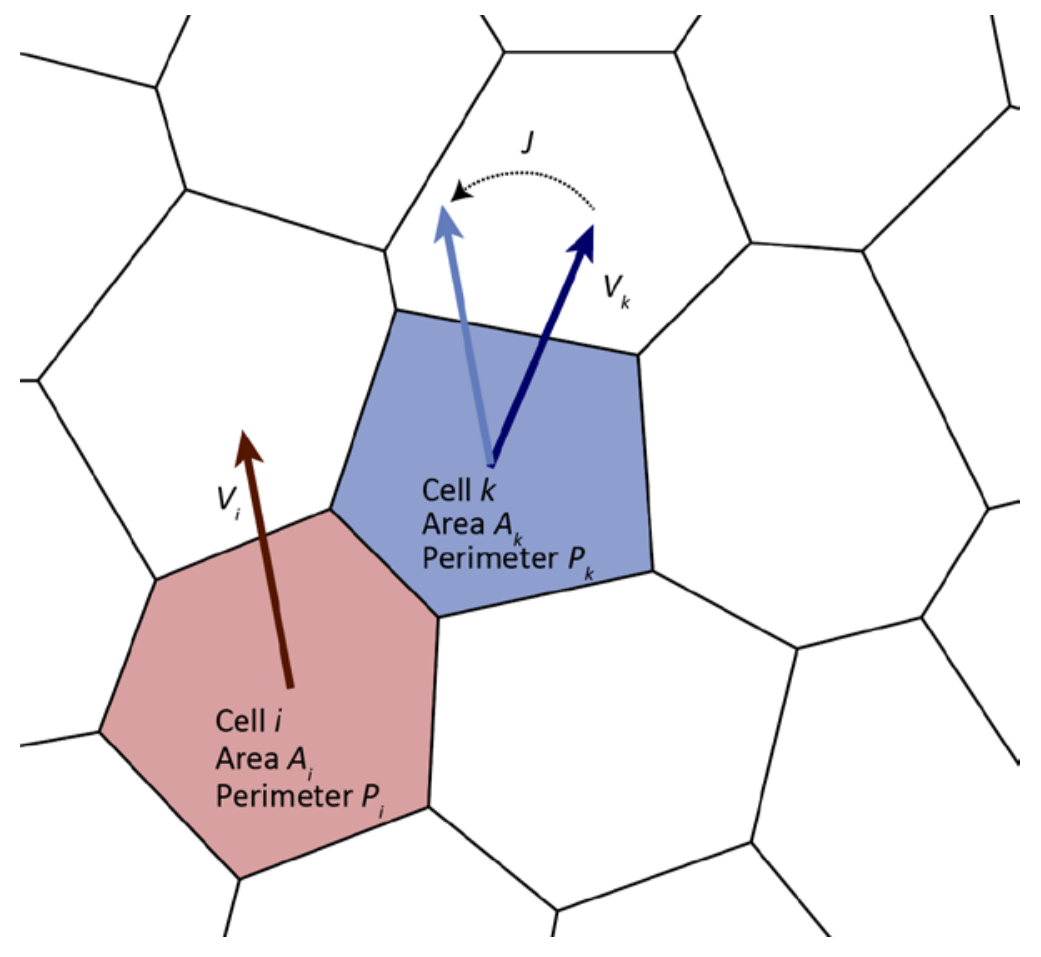

Figure Box 3. Illustration of Vertex and Self Propelled Voronoi Models. In the absence of self-propulsion, cell dynamics is determined by changes in cell area and perimeter. With selfpropulsion, two adjacent cells align their velocity vectors according to an alignment rate $J$. 


\section{FIGURES}

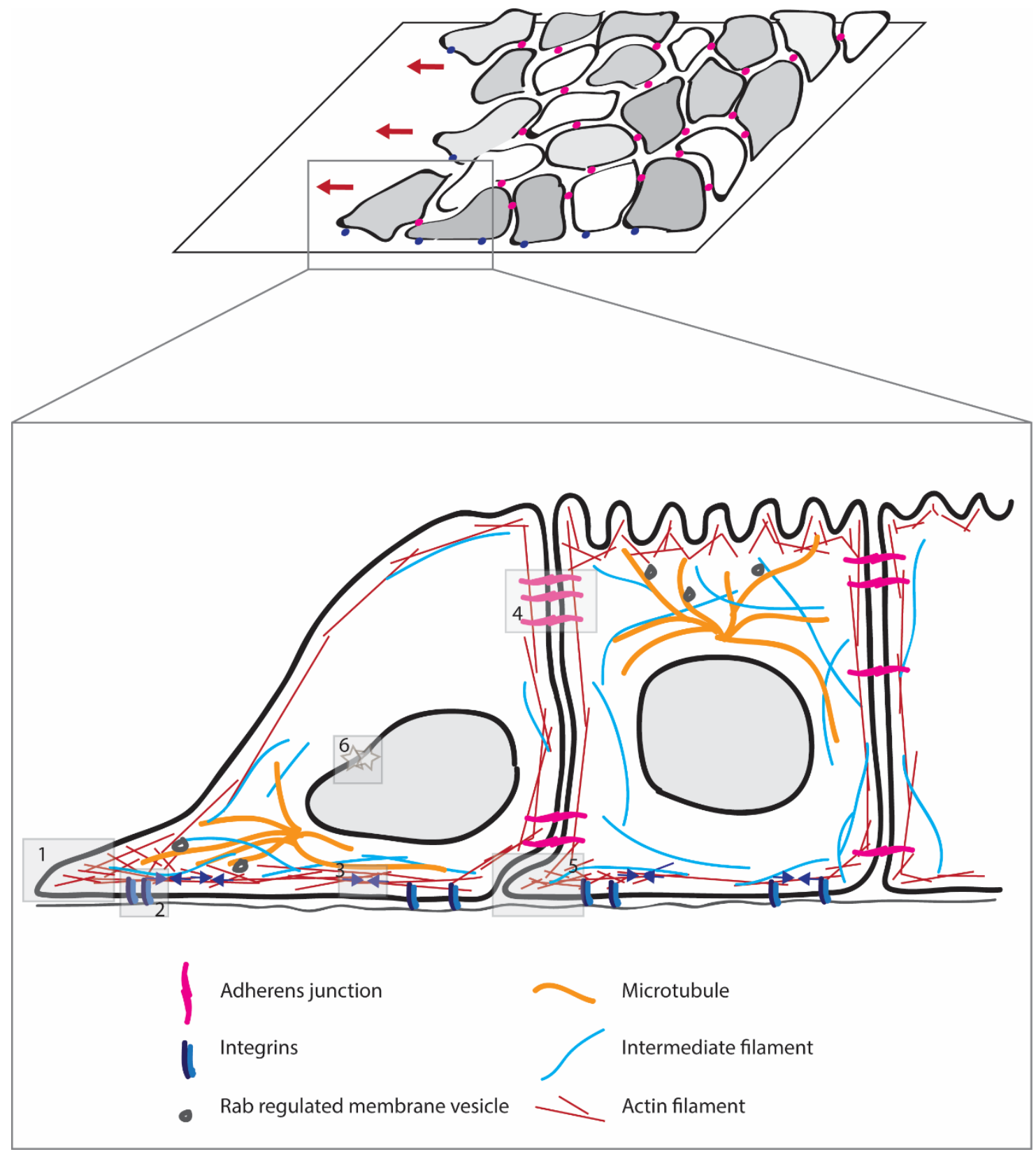

Figure 1. Subcellular determinants of cell dynamics. Top: a migrating epithelial monolayer, with blue and pink dots representing cell-matrix and cell-cell adhesions, respectively. Bottom: Image representation of a migratory cell (left) and a more typical epithelial cell (mid-right) with key features highlighted. 1) An actin rich protrusion - often termed a lamellipod, 2) An integrin mediated adhesion - often termed a focal adhesion, 3) An actomyosin contractile unit, 4) An adherens junction containing cadherins (in pink), 5) A 'cryptic' lamellipod that can enable the migration of the epithelial cell to the left while retaining adherens junctions, 6) Deformation of the nucleus during cell migration. 
a
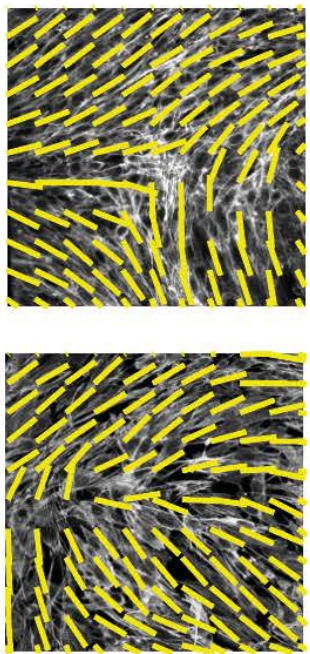

$100 \mu \mathrm{m}$
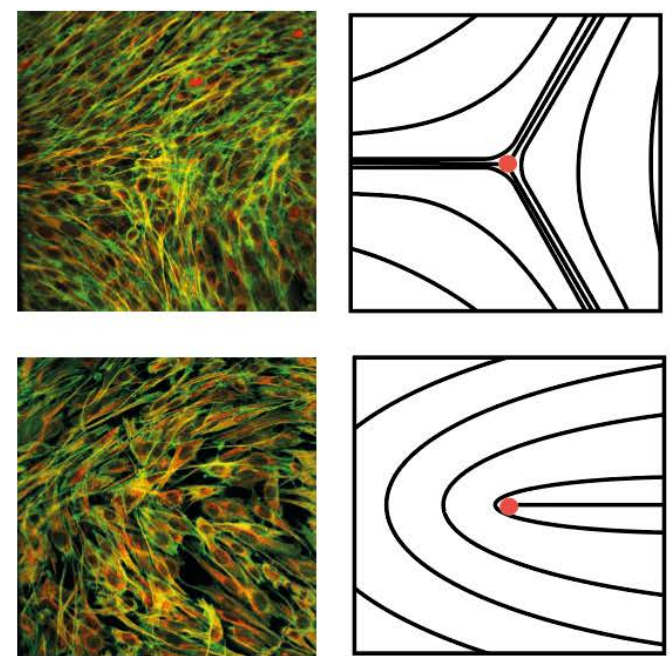

actin/tubulin

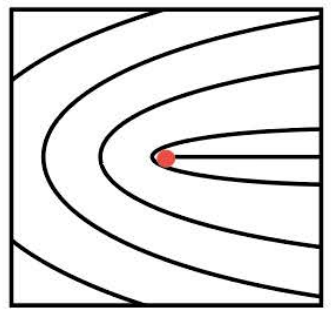

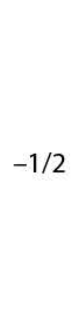
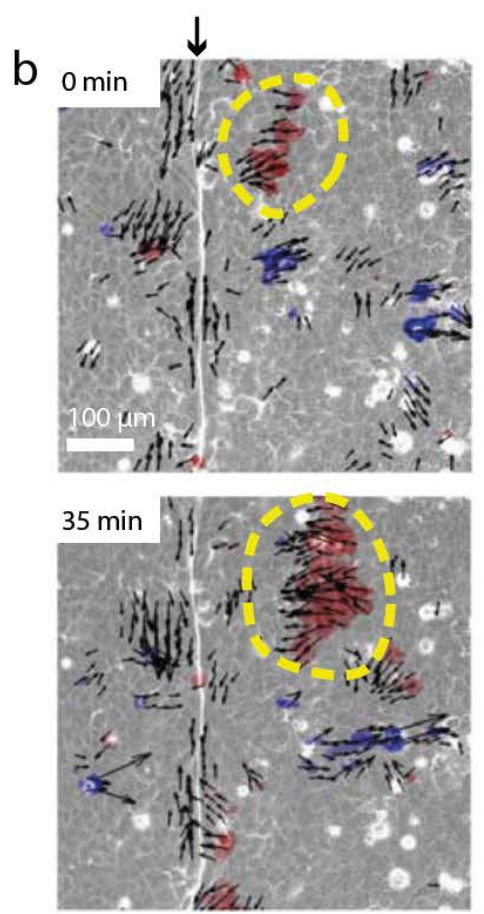

C

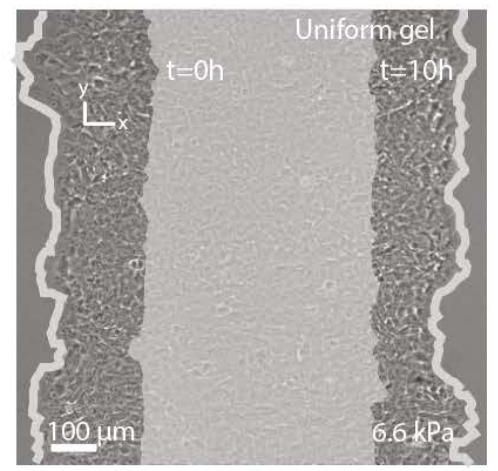

$$
\text { SOFT }
$$

STIFF
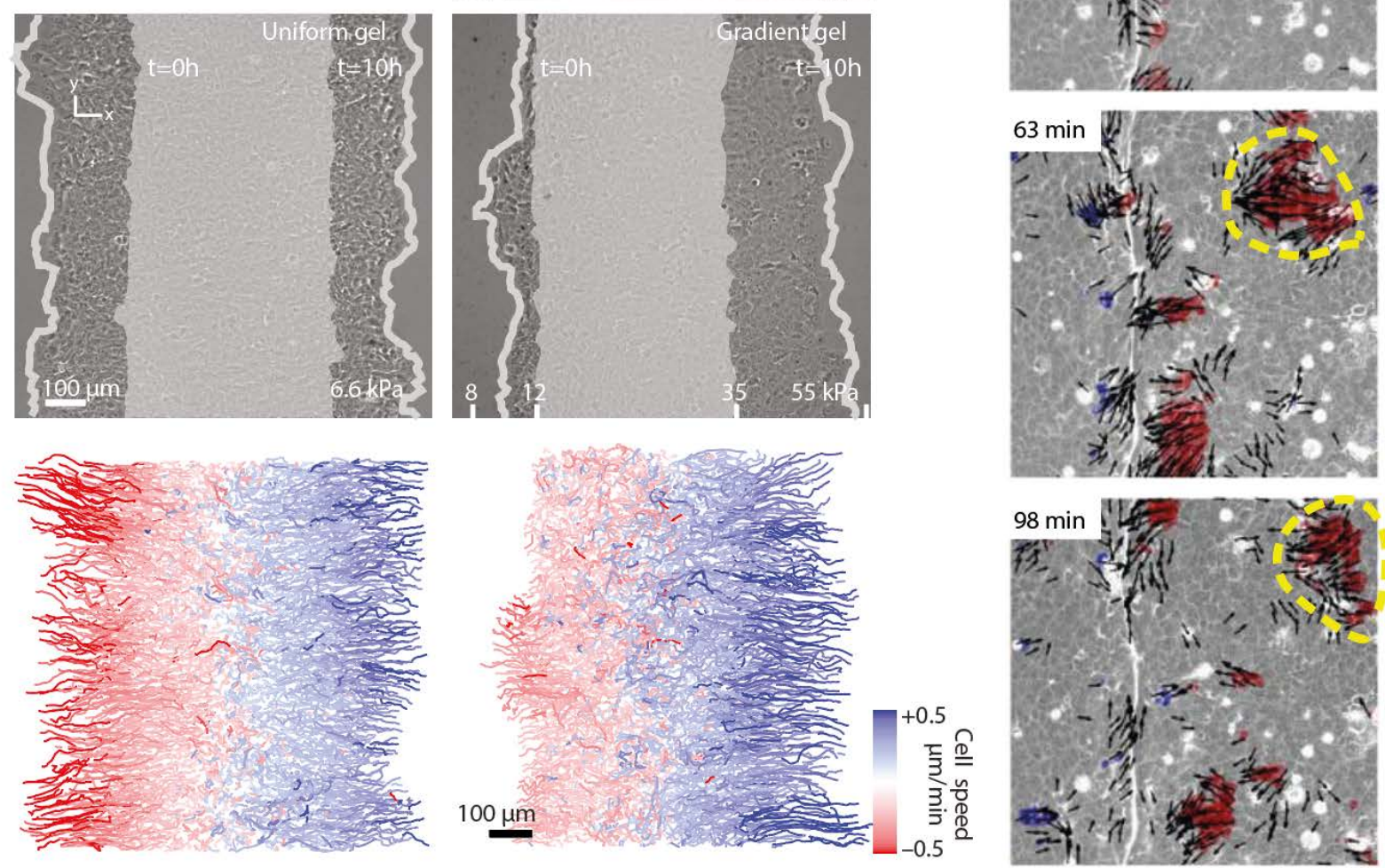

Figure 2. Examples of emergent phenomena in cell monolayers. (a) Topological defects. Left: orientation field superimposed on actin fluorescence images of a monolayer of NIH 3T3 mouse embryo fibroblasts. Centre: same area where actin and tubulin have been labelled. Right: schematics of the $+1 / 2$ and $-1 / 2$ defects. Adapted from reference 2. (b) Propagation of mechanical waves after a collision between monolayers expressing Eph and ephrin (black arrow indicates point of collision). Thresholded velocity fields superimposed on phase-contrast images. Dashed yellow lines highlight clusters of high forward velocity propagating backwards at different times. Arrows 
indicate the velocity vector. Scale bar is $100 \mu \mathrm{m}$. Adapted from reference ${ }^{78}$. (c) Collective sensing of a rigidity gradient by MCF10A cells. Left: a representative cell cluster expanding on a soft uniform gel of $6.6 \mathrm{kPa}$. The gray transparent area indicates initial cluster position ( $\mathrm{t}=0$ hours), and the phase-contrast image shows the cluster at 10 hours. Gray lines indicate cluster edges at 10 hours. Right: example of a cell cluster expanding on a gradient gel. Gel stiffness increases toward the right of the panel. Numbers at the bottom indicate Young's modulus. Bottom: individual cell trajectories corresponding to the experiments displayed in the top panels. Color coding indicates mean cell speed for every track. Adapted from reference 4. 

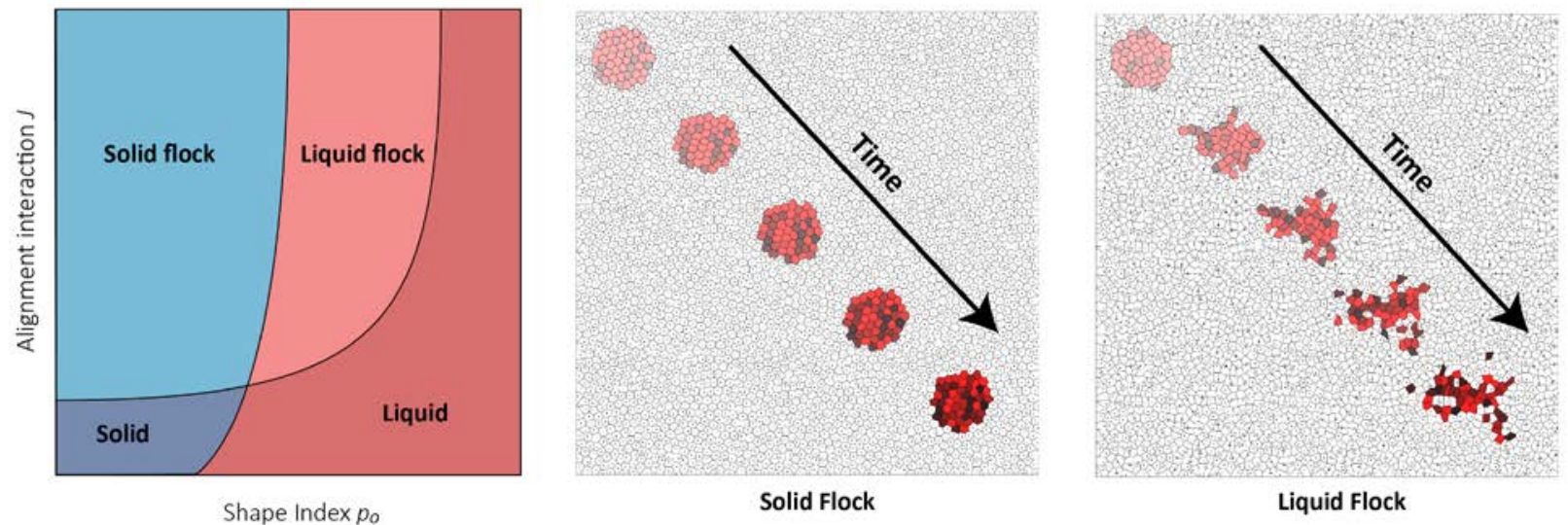

Figure 3: State diagrams of cell monolayers. Dynamic states of a cell monolayer. (a) State diagram depicting cell states as a function of the shape index and the alignment rate. Adapted from references ${ }^{86,87}$. (b) Time evolution of a cell cluster in a flocking solid state. Cells comprising the initial cluster move cohesively without neighbor exchanges. (c) Time evolution of a cell cluster in a flocking liquid state. Cells comprising the initial cluster drift with frequent neighbor exchanges. Images $\mathbf{b}$ and $\mathbf{c}$ are courtesy of R. Cerbino and F. Giavazzi. 